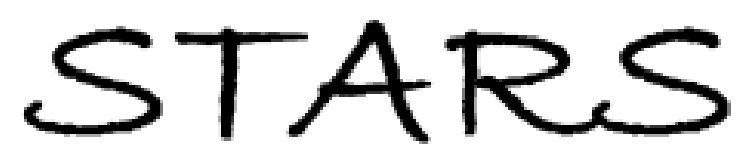

University of Central Florida

STARS

Faculty Bibliography 2000s

Faculty Bibliography

$1-1-2009$

\title{
Negative A-plates for broadband wide-view liquid crystal displays
}

Meizi Jiao

University of Central Florida

Sebastian Gauza

University of Central Florida

Yan Li

University of Central Florida

Jin Yan

University of Central Florida

Shin-Tson Wu

University of Central Florida

See next page for additional authors

Find similar works at: https://stars.library.ucf.edu/facultybib2000

University of Central Florida Libraries http://library.ucf.edu

This Article is brought to you for free and open access by the Faculty Bibliography at STARS. It has been accepted for inclusion in Faculty Bibliography 2000s by an authorized administrator of STARS. For more information, please contactSTARS@ucf.edu.

\section{Recommended Citation}

Jiao, Meizi; Gauza, Sebastian; Li, Yan; Yan, Jin; Wu, Shin-Tson; and Chiba, Tsuyoshi, "Negative A-plates for broadband wide-view liquid crystal displays" (2009). Faculty Bibliography 2000s. 1671.

https://stars.library.ucf.edu/facultybib2000/1671

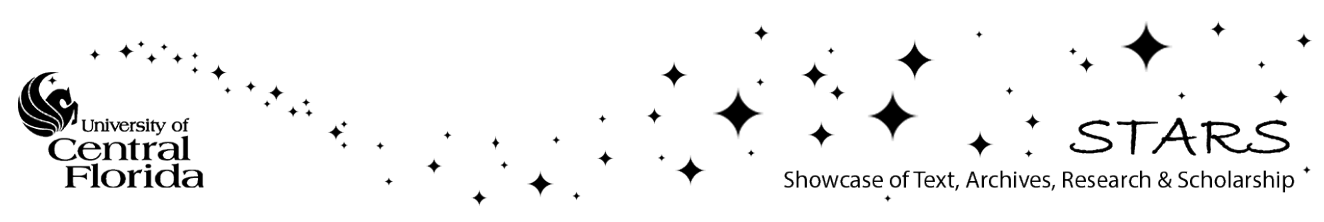




\section{Authors}

Meizi Jiao, Sebastian Gauza, Yan Li, Jin Yan, Shin-Tson Wu, and Tsuyoshi Chiba 


\section{Negative $A$-plates for broadband wide-view liquid crystal displays}

Cite as: Appl. Phys. Lett. 94, 101107 (2009); https://doi.org/10.1063/1.3097247

Submitted: 09 February 2009 . Accepted: 17 February 2009. Published Online: 10 March 2009

Meizi Jiao, Sebastian Gauza, Yan Li, Jin Yan, Shin-Tson Wu, and Tsuyoshi Chiba

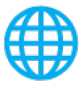

\section{ARTICLES YOU MAY BE INTERESTED IN}

Electro-optics of polymer-stabilized blue phase liquid crystal displays

Applied Physics Letters 94, 101104 (2009); https://doi.org/10.1063/1.3097355

Extraordinarily high-contrast and wide-view liquid-crystal displays

Applied Physics Letters 86, 121107 (2005); https://doi.org/10.1063/1.1887815

Tunable-focus flat liquid crystal spherical lens

Applied Physics Letters 84, 4789 (2004); https://doi.org/10.1063/1.1760226

\section{Applied Physics Letters}

Mid-IR and THz frequency combs special collection

Read Now! 


\section{Negative A-plates for broadband wide-view liquid crystal displays}

Meizi Jiao, ${ }^{1}$ Sebastian Gauza, ${ }^{1}$ Yan Li, ${ }^{1}$ Jin Yan, ${ }^{1}$ Shin-Tson Wu, ${ }^{1, a)}$ and Tsuyoshi Chiba ${ }^{2}$

${ }^{1}$ College of Optics and Photonics, University of Central Florida, Orlando, Florida 32816, USA

${ }^{2}$ Nitto Denko Corporation, Onomichi, Hiroshima 722-0212, Japan

(Received 9 February 2009; accepted 17 February 2009; published online 10 March 2009)

Negative $A$-plates fabricated by mechanically stretching polystyrene are studied and their physical properties evaluated. A molecular model is developed to explain the underlying physical mechanisms. An example of a broadband wide-view liquid crystal display including a negative A-plate for phase compensation is illustrated. () 2009 American Institute of Physics.

[DOI: $10.1063 / 1.3097247$ ]

High contrast ratio and wide viewing angle are critical requirements for direct view liquid crystal displays (LCDs). Various optical compensation schemes with phase-matched films have been developed to widen the viewing angle of LCDs. ${ }^{1-5} A$-plate, whose optic axis is located in the plane parallel to film surface, has been widely used in wide-view LCDs. Although positive and negative $A$-plates are usually equivalent to each other, in some applications negative $A$-plate has its unique functions. ${ }^{5-8}$ For instance, a negative $A$-plate together with a positive $A$-plate reduce the phase mismatch at oblique viewing angle and thereby improve the off-axis image quality ${ }^{5,7}$ Besides, the adoption of negative $A$-plate makes it easier to achieve wide view and broad bandwidth. ${ }^{6}$ Negative $A$-plates have found emerging applications in transmissive and transflective LCDs.

For large LCD panels, the compensation films are commonly made by stretching or compressing polymers at their glassy states. The stretching method offers good uniformity, low cost, and high yield. ${ }^{9,10}$ While the fabrication technique for $C$-plates and positive $A$-plates is relatively mature, negative $A$-plates have not been well explored. So far, several approaches have been proposed, such as photoinduced polymerization ${ }^{11}$ and self-assembled lyotropic liquid crystal films using coating or printing method. ${ }^{12,13}$ Both approaches are effective for obtaining a large negative in-plane birefringence but neither is ready for widespread applications.

In this letter, we fabricated negative $A$-plates by stretching polystyrene (PSt) around its glass-transition temperature and measured its physical properties, such as refractive indices and birefringence dispersion. We also developed a molecular model to explain why the stretched polystyrene exhibits a negative birefringence. Finally, a broadband wideview LCD using the negative $A$-plate is proposed and the suppression of light leakage over visible spectrum is demonstrated.

Negative $A$-plates can be obtained by stretching negative birefringence materials, such as PSt. The molecular structure of PSt is shown as follows:

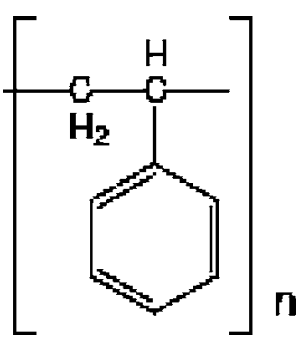

We have modeled a core structure of PSt using HYPERCHEM molecular modeling software. A semiempirical method of modified neglect of diatomic overlap was used with Polak-Ribiere geometry optimization algorithm. As a result, a single molecular core with side phenyl units was calculated in vасио to its possibly lowest energy gradient, which suggests most possible configuration of the molecule. Figure 1 shows molecular configuration of PSt optimized to total energy gradient of $10^{-5} \mathrm{kcal} /(\mathrm{mol} \AA)$ calculated as a rootmean-square value. The energy gradient is the rate of change (first derivative) of total energy with respect to displacement

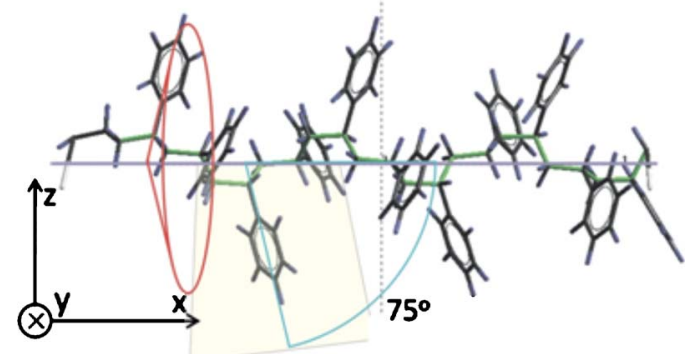

(a)

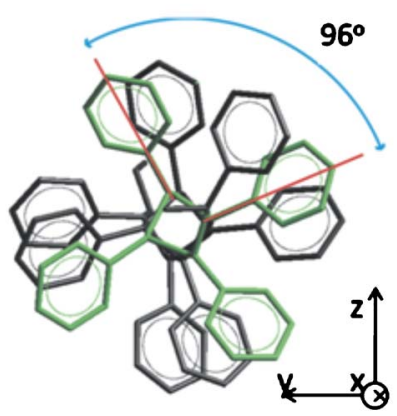

(b)

FIG. 1. (Color online) Molecular conformation of stretched PSt. (a) Side view and (b) front view.

${ }^{a)}$ Electronic mail: swu@ mail.ucf.edu. 
of each atom in the $x, y$, and $z$ directions. Here, the mechanical stretching occurs along $x$-axis.

As shown in Fig. 1(a), an imaginary long molecular axis is formed along $x$-axis with all the backbone carbon atoms surrounding it. The side phenyl ring is connected to every other carbon, with a $75^{\circ}$ angle phenyl ring plane and the imaginary long axis. The birefringence of PSt mainly originates from the conjugated electrons of side phenyl rings. Since $45^{\circ}<75^{\circ}<90^{\circ}$, after projection the refractive index along the long molecular axis $\left(n_{x}\right)$ is smaller than the perpendicular components $\left(n_{y}\right.$ and $\left.n_{z}\right)$. Moreover, the model reveals that the distribution of side phenyl rings is very uniform. Figure 1(b) depicts the Newman projection of the PSt molecule. For simplicity, the hydrogen atoms are neglected in the drawing. The angle between each pair of neighboring phenyl rings is $96^{\circ}$, which means that every 15 side-chain phenyl rings complete a cycle. Within each cycle, the phenyl rings are evenly separated. Therefore, the refractive indices in $y-z$ plane are the same at all directions, i.e., $n_{y}=n_{z}$. This is the definition of a negative $A$-plate whose refractive indices satisfy following order: $n_{x}<n_{y}=n_{z}$.

The above description of molecular modeling unfolds the local property of a PSt molecule. At a glassy state, the molecules are randomly oriented and thereby the material is isotropic. By stretching the PSt in the $x$-axis, the molecules tend to align with their long molecular axis along $x$ direction. In this way, a negative $A$-plate with $n_{x}<n_{y}=n_{z}$ is obtained.

Based on the above model, the basic criteria for obtaining a negative $A$-plate are twofold: (1) to have side chains that contribute more to the refractive index at the direction perpendicular to the long molecular axis and (2) to have a better orientation of the long molecular axis along the stretching direction.

We fabricated negative $A$-plates by stretching PSt films around its glass-transition temperature $\left(T_{g} \sim 100{ }^{\circ} \mathrm{C}\right)$. The refractive indices were measured by an ellipsometry to be $n_{x}=1.5805$ and $n_{y}=n_{z}=1.5812$ at $\lambda \sim 590 \mathrm{~nm}$. Meanwhile, the transmittance of a setup with negative $A$-plate orientated at $45^{\circ}$ with respect to the optic axes of the crossed polarizers was measured using a spectrophotometer. The wavelength dependent birefringence was obtained through the normalized transmittance. ${ }^{14}$

Figure 2 depicts the birefringence dispersion of the negative $A$-plate. Black curve is the measured data and red denotes the fitting curve based on the birefringence dispersion equation $\Delta n=G \lambda^{2} \lambda^{* 2} /\left(\lambda^{2}-\lambda^{* 2}\right),{ }^{15,16}$ where $G$ is a proportionality constant and $\lambda^{*}$ is the mean resonance wavelength. The fitting curve with $\lambda^{*}=177.7 \mathrm{~nm}$ and $G=1.775$ $\times 10^{-5} \mathrm{~nm}^{-2}$ agrees very well with the experimental results. For the PSt negative $A$-plate, $\lambda^{*}$ represents the resonance wavelength of the conjugated electrons of the side phenyl rings. A shorter $\lambda^{*}$ leads to a weaker dispersion in the visible spectral region. ${ }^{17}$

Negative $A$-plates have many applications in transmissive LCDs, ${ }^{5}$ wide-view and broadband circular polarizers, ${ }^{6}$ and simple compensation scheme of transflective LCDs. ${ }^{8}$ Here, we propose a simple compensation configuration using our negative $A$-plate to achieve a broadband wide-view multidomain alignment (MVA) LCD.

The inset in Fig. 3 shows the proposed compensation scheme. The LCD works under a crossed linear polarizers system. The transmission axes of polarizer and analyzer are

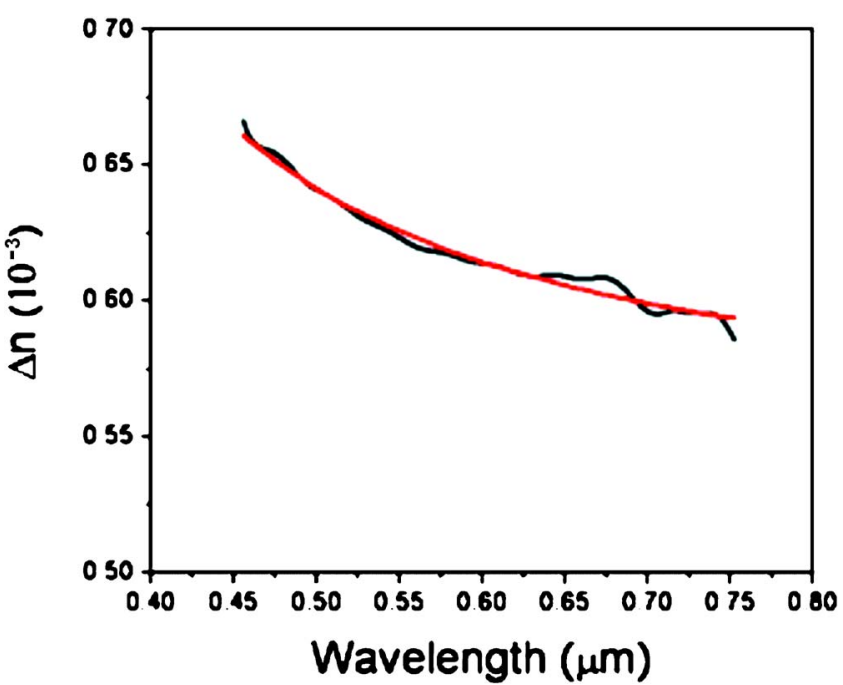

FIG. 2. (Color online) Wavelength dependent birefringence of the stretched $A$-plate. Black curve stands for the experimental data and red curve for the fitting results.

at $-45^{\circ}$ and $45^{\circ}$, respectively. Three compensation films, a negative $C$-plate, a negative $A$-plate, and a positive $A$-plate, are laminated successively on the LC cell. The optic axes of the two A-plates are both parallel to the transmission axis of the analyzer.

The conventional compensation scheme with a negative $C$-plate and a positive $A$-plate is commonly used for MVA LCD. ${ }^{5}$ It offers wide view for an optimized wavelength but not for the whole visible spectral region. With only one negative $A$-plate added, our compensation is able to suppress the light leakage over the whole visible spectrum and lead to a wide view under white light. $C$-plate usually has a weaker dispersion than $\mathrm{LC}$, so we intentionally designed the $C$-plate to have a larger phase retardation $d \Delta n$. In this way, LCs phase retardation change under different wavelengths can be compensated by the phase retardation change of the negative $C$-plate. Overall speaking, the $\mathrm{LC}$ and negative $C$-plate work together as an effective dispersion-free negative $C$-plate. Likewise, the dispersions of the negative and positive $A$-plates are canceled by each other. The dispersion of our PSt negative $A$-plate is relatively weak. Positive $A$-plates with stronger dispersion characteristic can be easily obtained

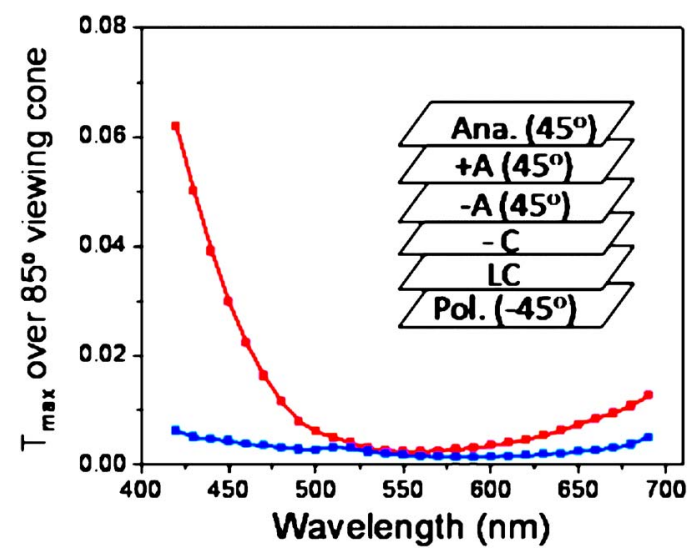

FIG. 3. (Color online) Comparison of the maximum light leakage over $85^{\circ}$ viewing cone between the conventional (red curve) and the proposed (blue curve) compensation schemes for a MVA LCD. The inset illustrates the proposed compensation configuration. 
from polycarbonate or polysulfone films. ${ }^{10}$ With optimized film thicknesses, the two $A$-plates together behave as a dispersion-free negative $A$-plate. Theoretically, a perfect dark state can be obtained with this compensation configuration.

We calculate the LC director's distribution by finite element method $^{18}$ and optical properties by extended $2 \times 2$ Jones matrix methods. ${ }^{19,20}$ The LC used in calculation is MLC-6608 with parameters as follows: $K_{11}=16.7 \mathrm{pN}, K_{22}$ $=7.3 \mathrm{pN}, K_{33}=18.1 \mathrm{pN}$, birefringence $\Delta n=0.0836$ at $\lambda$ $=550 \mathrm{~nm}$, dielectric anisotropy $\Delta \varepsilon=-4.2$, and rotational viscosity $\gamma_{1}=186 \mathrm{mPa}$. The LC cell gap is $4 \mu \mathrm{m}$. The phase retardation $d \Delta n$ at $\lambda=550 \mathrm{~nm}$ is $370,491.4$, and $355 \mathrm{~nm}$ for negative $C$-plate, negative $A$-plate, and positive $A$-plate, respectively. The dispersion of negative $A$-plate is based on the experimental data of our PSt negative $A$-plate. The dispersions of other layers are all based on data from commercially available products.

Figure 3 compares the maximum light leakage over $85^{\circ}$ viewing cone of the conventional and proposed compensation configurations. Red curve stands for the abovementioned conventional compensation scheme while the blue for the proposed one. The light leakage is suppressed significantly over the entire visible spectra. This would lead to a broadband and wide-view LCD with good color saturation.

In conclusion, our molecular model elucidates the physical mechanisms why the stretched PSt behaves like a negative $A$-plate. The side chain conjugation and its orientation angle, and main chain alignment (through stretching strength) affect the refractive indices significantly. A larger negative birefringence would enable a thinner film to be used. This understanding of negative $A$-plate will enable better optical films to be developed which will further enhance the LCD performances.
The authors are indebted to Dr. Zhibing Ge and Professor Jiyu Fang for useful technical discussions and Chi-Mei Optoelectronics (Taiwan) for financial support.

${ }^{1}$ K. Ohmuro, S. Kataoka, T. Sasaki, and Y. Koike, SID Int. Symp. Digest Tech. Papers 33, 845 (1997).

${ }^{2}$ J. Chen, K. H. Kim, J. J. Jyu, J. H. Souk, J. R. Kelly, and P. J. Bos, SID Int. Symp. Digest Tech. Papers 29, 315 (1998).

${ }^{3}$ R. Lu, X. Zhu, S. T. Wu, Q. Hong, and T. X. Wu, J. Disp. Technol. 1, 3 (2005)

${ }^{4}$ Q. Hong, T. X. Wu, X. Zhu, R. Lu, and S. T. Wu, Appl. Phys. Lett. 86, 121107 (2005).

${ }^{5}$ X. Zhu, Z. Ge, and S. T. Wu, J. Disp. Technol. 2, 2 (2006).

${ }^{6}$ Z. Ge, M. Jiao, R. Lu, T. X. Wu, S. T. Wu, W. Y. Li, and C. K. Wei, J. Disp. Technol. 4, 129 (2008).

${ }^{7}$ C. H. Lin, Appl. Phys. Lett. 90, 151112 (2007).

${ }^{8}$ J. Matsushima, S. Uehara, and K. Sumiyoshi, Proceedings of the International Display Workshops, 2007 (unpublished), p. 1511.

${ }^{9}$ H. Yoshimi, S. Yano, and Y. Fujimura, SID Int. Symp. Digest Tech. Papers 33, 862 (2002).

${ }^{10}$ S. T. Wu, Mater. Chem. Phys. 42, 163 (1995).

${ }^{11}$ T. Sergan, M. Sonpatki, J. Kelly, and L. C. Chien, Mol. Cryst. Liq. Cryst. 359, 245 (2001).

${ }^{12}$ P. I. Lazarev, A. A. Manko, S. V. Remizov, S. P. Palto, and A. P. Lazarev, Proc. SPIE 6286, 9 (2006).

${ }^{13}$ P. Lazarev, A. Manko, I. Kasianova, and S. Palto, Proc. SPIE 6637, 66370J (2007)

${ }^{14}$ S. T. Wu, U. Efron, and L. D. Hess, Appl. Opt. 23, 3911 (1984).

${ }^{15}$ S. T. Wu, Phys. Rev. A 33, 1270 (1986).

${ }^{16} \mathrm{~S}$. T. Wu, C. S. Wu, M. Warenghem, and M. Ismaili, Opt. Eng. (Bellingham) 32, 1775 (1993).

${ }^{17}$ J. Li and S. T. Wu, J. Appl. Phys. 95, 896 (2004).

${ }^{18}$ Z. Ge, T. X. Wu, R. Lu, X. Zhu, Q. Hong, and S. T. Wu, J. Disp. Technol. 1, 194 (2005)

${ }^{19}$ A. Lien, Appl. Phys. Lett. 57, 2767 (1990).

${ }^{20}$ Z. Ge, T. X. Wu, X. Zhu, and S. T. Wu, J. Opt. Soc. Am. A-Opt. Image Sci. Vis 22, 966 (2005) 